\title{
Endothelial dysfunction and tendinopathy: how far have we come?
}

\author{
R. Papalia $\cdot$ L. Moro $\cdot$ F. Franceschi $\cdot$ \\ E. Albo · S. D'Adamio • A. Di Martino • \\ G. Vadalà $\cdot$ C. Faldini $\cdot$ V. Denaro
}

Received: 4 July 2013/Accepted: 17 July 2013/Published online: 2 August 2013

(C) Istituto Ortopedico Rizzoli 2013

\begin{abstract}
Symptomatic tendon tears are one of the most important causes of pain and joint dysfunction. Among the intrinsic causes, vascularization recently gained a major role. Endothelial function is indeed a key factor, as well as vascular tone and thrombotic factors, in the regulation of vascular homeostasis and the composition of vascular wall. In this review, we studied systematically whether there is a relationship between endothelial dysfunction and tendinopathy. A literature search was performed using the isolated or combined keywords endothelial dysfunction and tendon,' 'nitric oxide (NO) and tendinopathy,' and 'endothelial dysfunction in tendon healing.' We identified 21 published studies. Of the selected studies, 9 were in vivo studies, 2 focusing on animals and 7 on humans, while 12 reported about in vitro evaluations, where 7 were carried out on humans and 5 on animals. The evidence about a direct relationship between tendinopathy and endothelial dysfunction is still poor. As recent studies have shown,
\end{abstract}

R. Papalia · F. Franceschi · E. Albo · S. D'Adamio .

A. Di Martino $(\bowtie) \cdot$ G. Vadalà · V. Denaro

Department of Orthopaedic and Trauma Surgery, CIR,

Center of Integrated Research, University Campus Bio-Medico of Rome, Via Alvaro del Portillo, 200, 00128 Rome, Italy

e-mail: dimartino.cbm@gmail.com

L. Moro

Department of Geriatrics, CIR, Center of Integrated Research, University Campus Bio-Medico of Rome, Rome, Italy

C. Faldini

Department of Rizzoli-Sicilia, Orthopaedic Service,

The Rizzoli Institute and University of Bologna, Bologna, Italy there is no significant improvement in clinical and functional assessments after treatment with $\mathrm{NO}$ in patients suffering from tendinopathy in different locations. No significant differences were identified in the outcomes reported for experiment group when compared with controls treated with conventional surgical procedures or rehabilitation programs. Nitric oxide could be a marker to quantify the response of the endothelium to mechanical stress or hypoxia indicating the final balance between vasodilatating and vasoconstricting factors and their effects, but more ad stronger evidence is still needed to fully support this practice.

Keywords Tendinopathy $\cdot$ Endothelial dysfunction . NO $\cdot$ Nitric oxide

\section{Introduction}

Musculoskeletal injuries related to overuse are common in tendons of the rotator cuff, lateral epicondyle of the elbow, patella, and Achilles [11]. Essentially, tendinopathy is a condition of impaired healing response to stress, in which tenocytes degeneration and collagen fibers disruption weaken the overall tendon structure. Evidences showed how load and microinjury of the Achilles tendon structure from continuous involvement can result in a partially damaged tendon where the injured portions unload and the remaining intact portions consequently bear most of the force applied [36]. Also the optimum treatment of complete rupture of Achilles tendon has not been established yet, the most frequently ruptured tendon in the human body $[22,35]$.

Symptomatic rotator cuff tears cause severe pain and disability of the shoulder [7, 14, 23, 29, 32]. Emerging 
concepts highlight that cuff tendinopathy, and eventually tendon tears, could be the result of an abnormal inflammatory status and unbalanced enzymatic cascades within the tissue involved [1, 4]. Extrinsic causes such as repetitive microtrauma in overhead athletes and subacromial impingement in older individuals also play a role in the pathogenesis of this multifactorial condition [2,16], due to the altered cellular metabolism in association with aging processes (degeneration [1], abnormal apoptosis [2], and chronic inflammation [8]). Based on the concept of 'critical area' of the supraspinatus described by Codman, hypovascularization may predispose to the development of tendinopathy [3]. As shown by the analysis of microvascular systems carried out in recent years [37], endothelial function is regulated by a finely balanced equilibrium between vasorelaxing and vasoconstricting mediators [10, 28]. In this context, nitric oxide (NO) has been investigated as having an important role in these processes: $\mathrm{NO}$ is an endothelium-derived vasodilator factor which reduces the release of vasoconstrictor reactive oxygen species [28], reacts with toxic superoxides, and stimulates the production of peroxynitrites, free radicals, eventually predisposing to endothelial dysfunction.

We systematically review the published literature regarding the association between endothelial dysfunction and rotator cuff disease to try and answer the following questions: (1) Which factors are involved in endothelial dysfunction and what is the correlation between this condition and tendinopathy? (2) How is it possible to diagnose an endothelial dysfunction? (3) Can our understanding be helpful to manage and prevent rotator cuff disorders?

\section{Methods}

Search and study selection

We searched for relevant published studies in Pubmed, Medline, Ovid, Google Scholar, and Embase databases using the isolated and combined keywords 'endothelial dysfunction and tendon,' 'NO and tendinopathy,' and 'endothelial dysfunction in tendon healing,' with no limit for the year of publication (Fig. 1). We included studies in English, Spanish, French, and Italian, published in peerreviewed journals, reporting data on clinical and functional outcomes of patients with tendinopathy. Biomechanical reports, studies on animals, cadavers, in vitro or animal studies, and case reports were included. Literature reviews, technical notes, letters to editors, and instructional course were excluded. Two authors (RP and EA) independently assessed the full-text version of each publication, selecting on the basis of its content and excluding papers without the specific content. The reference lists of the selected articles were fully reviewed by hand to identify articles not included at the first electronic search. Considering all the journals, we first identified 46 articles coherent with the topic at hand. After primary selection excluding studies not reporting about tendon pathology, all the authors retrieved, reviewed, and discussed 24 articles, excluding 14 studies reporting outcomes of patients with tendon repair without specific discussion on the role of endothelial dysfunction in the pathogenesis of tendinopathy. At the end of the study selection process, 21 relevant publications were included. Two authors (RP and EA) assessed and extracted data independently from each article.
Fig. 1 Process of inclusion of the studies

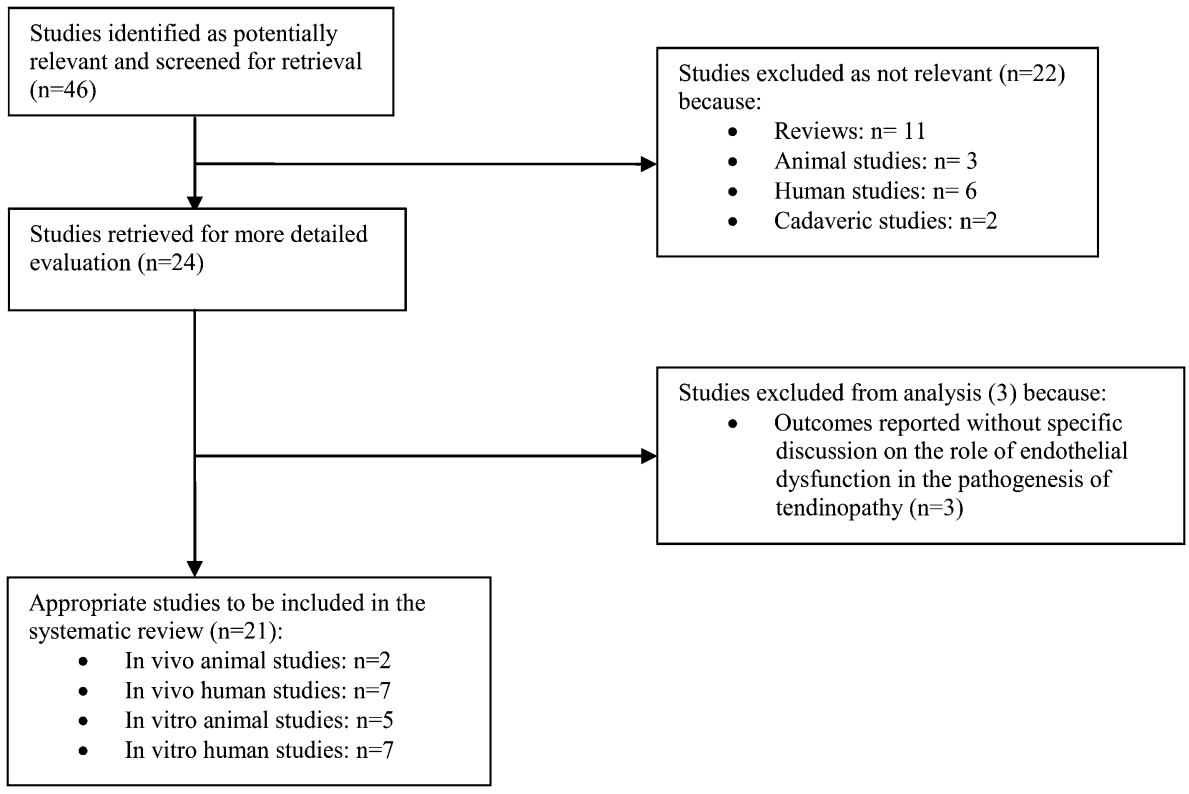




\section{Results}

We selected 21 studies: 9 were in vivo studies, 2 focusing on animals and 7 on humans, while 12 reported about in vitro evaluations, where 7 were carried out on humans and 5 on animals.

In vivo animal studies (Table 1)

Szomor et al. [33] recorded a fourfold increase in the expression of endothelial nitric oxide synthase (eNOS) and inducible nitric oxide synthase (iNOS), and an almost 3 time increase in neuronal nitric oxide synthase (nNOS) in supraspinatus tendons subjected to an overuse protocol, and a basal expression of these isoforms in control tendons.

The role of NO in healing process was investigated by Xia et al. [39] which, at 7 days from Achilles tendon surgery, observed that the healing process in iNOS gene knockout (iNOS -/-) mice was comparable to that observed in wild-type (iNOS +/+) mice. In cross-section analyses, the iNOS $-/-$ mice group treated with aminoguanidine (AG), a competitive nitric oxide synthase inhibitor, had a healing area $20 \%$ lower than that observed in the other tendons. On the other hand, the serum nitrate concentrations of both the iNOS $-1-$ mice group and the iNOS - /- mice with AG group were noticeably lower than that in iNOS $+/+$ mice group.

In vivo human studies (Table 2)

Administration of glyceryl topical trinitrate (GTN) for more than 3 months was shown to be effective in patients with supraspinatus tendinopathy, apparently because of the increased production of NO [26]. In a prospective randomized study, the application of GTN patches for 24 weeks markedly improved pain, range of motion, strength, impingement-related symptoms, and patient outcomes confirming that it is useful in this area.

At 12 and 24 weeks, chronic tendinopathy of the Achilles tendon appeared to benefit from topical glyceryl trinitrate patch in addition to rehabilitation treatment [25]. After 6 months of treatment, reduction in pain and improved outcomes were not significantly different when compared to controls following a standard exercise program [12].

Similar results were obtained by applying the GNT patches for chronic extensor tendinopathy of the elbow. Reduction in elbow pain with activity, decrease in epicondylar tenderness, and increase in wrist extensor mean peak force were shown at 6 months follow-up [27]. After 5 years, data lost significance and patient outcomes became comparable to those exclusively exercising [15].

Steunebrink et al. [31] recorded similar outcomes in patients with chronic patellar tendinopathy using a

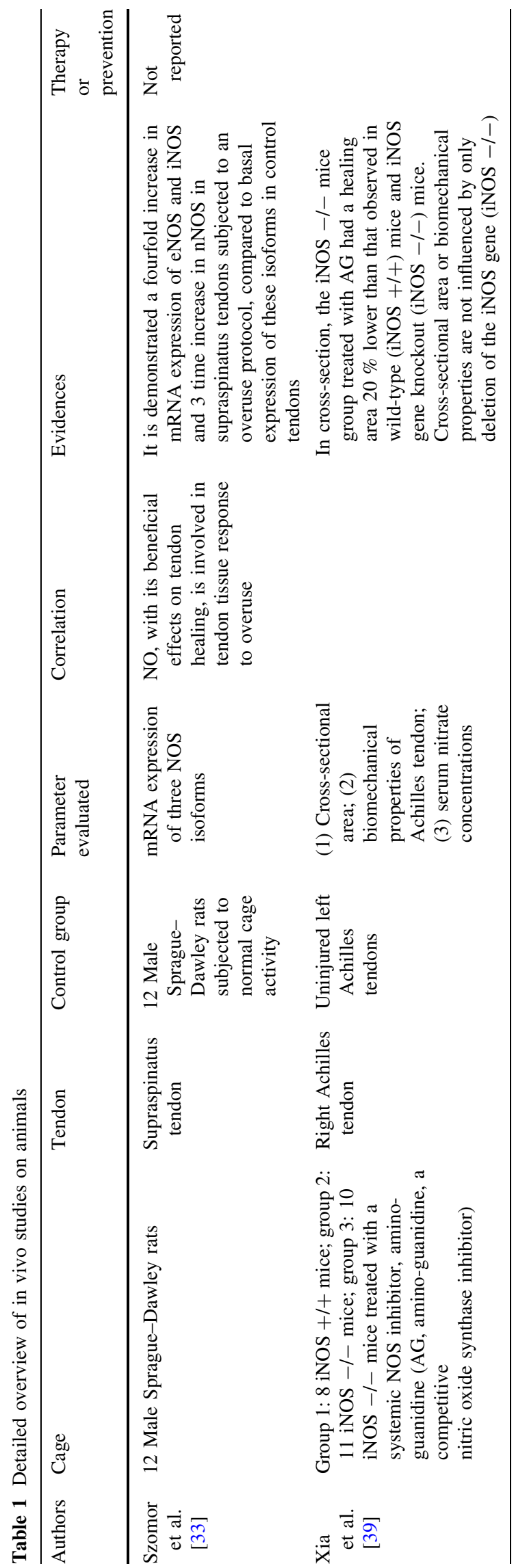




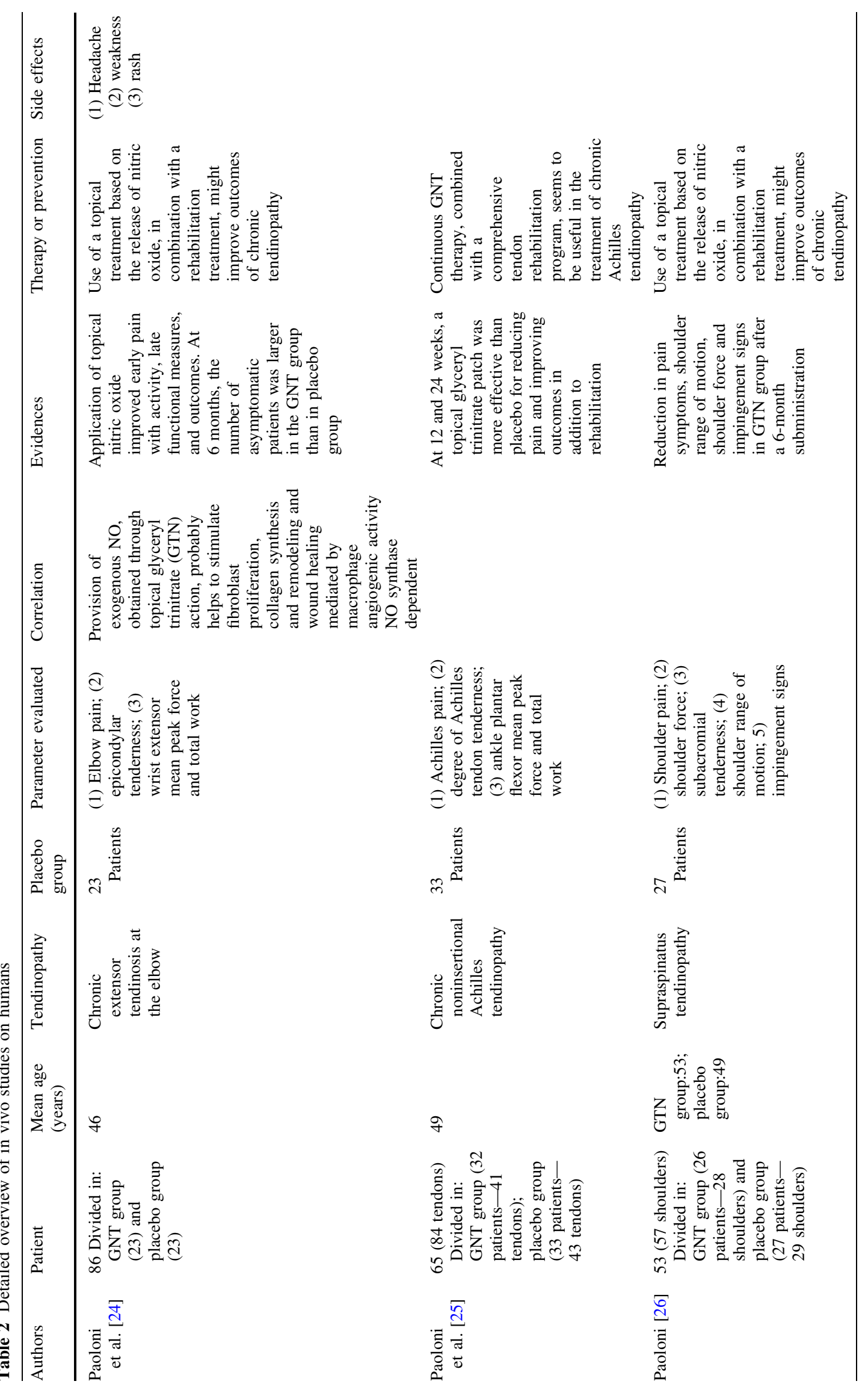




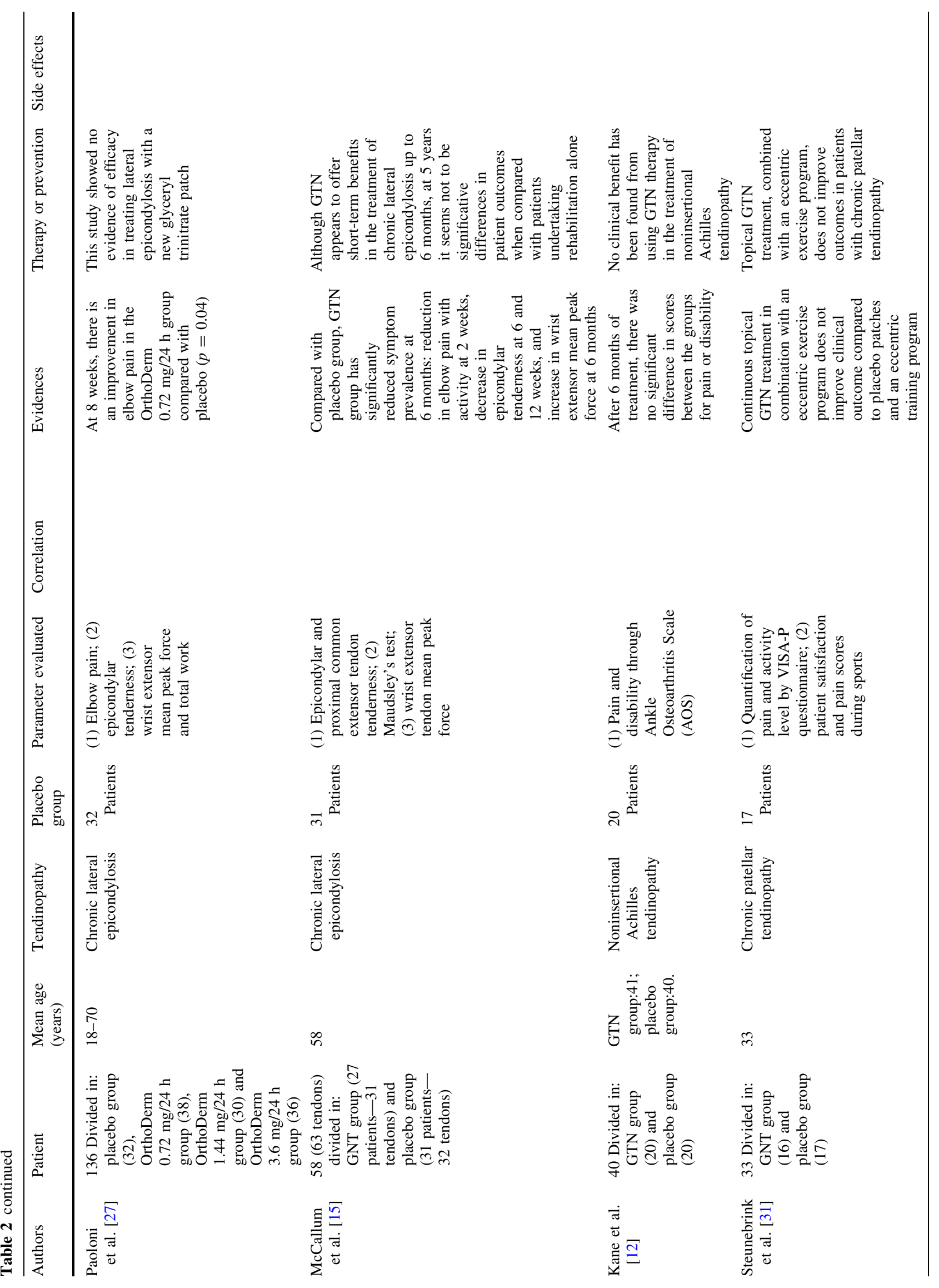


combination of GTN patches applications along with eccentric training in patients when compared to a control group who blindly applied a placebo patch while following the same physical rehabilitation program.

In vitro human studies (Table 3)

From the analysis of the nitric oxide synthase isoforms in 17 subacromial bursal samples, significant presence of eNOS and iNOS mRNA was found in all samples, and nNOS in 5 of them (29.4\%) [34].

In 14 patients undergoing rotator cuff repair, the stimulation of the tendon cells in culture with exogenous NO (SNAP) and iNOS gene carrier followed by enzymatic inhibition showed that low doses of NO positively influence the fibroblastic synthesis of collagen, while high doses inhibit it [38]. Murrel et al. [19] demonstrated that oxygen free radicals, whose values are strictly modulated by concentration of NO, may modulate the growth of fibroblasts in vitro, with increased cell density and cell size when the concentration of free radicals is low, and evidence of cellular damage when the concentration is high.

In patients with early-moderate tendinopathy of the supraspinatus tendon, inflammation may be dangerous, especially when mediated by mast cells. When mechanical stresses are applied, pro-inflammatory factors such as cytokines, oxygen free radicals, and proteases may induce tissue to a failed healing response which is considered to be the main pathogenetic factor in the development of tendon conditions. By the way, mast cell degranulation should release vasoactive and angiogenic factors contributing to the healing process; however, in severe tendinopathy, the concentration of inflammatory cells is high indeed while vascularization is shown to be insufficient to carry healing mediators on site [16].

In a study on 27 patients with subacromial impingement and partial to full thickness rotator cuff tears, the expression of HIF-1a, a transcription factor characteristic of the hypoxic environment, and Bnip3, a regulator of the HIF-1a proapoptotic protein, were analyzed in immunohistochemistry. The presence of fragmented DNA, common when apoptosis occurs, was often found local tissue samples demonstrating how this process is significantly increased in tendinopathy. Apoptosis involves fibroblasts or fibroblast-like cells, which ordinarily synthesize extracellular matrix, resulting in a hypoxic environment with impaired collagen synthesis. Therefore, hypoxia could regulate apoptosis by increasing its rate, especially in severe tendinopathy. The detection of increased hypoxic markers in the early stages of tendinopathy proved that hypoxia is involved in the regulation of apoptosis, probably as a consequence of the release of factors involved in reparative and degenerative pathways of the tendon [2].
Therefore, in hypoxic conditions, a dynamic balance between proapoptotic and anti-apoptotic mediators is crucial in the healing process [17].

Kane et al. [12] proved that neovascularization stimulation of wound fibroblasts and collagen synthesis was not found in patients exposed to GTN therapy. Also, the modulation of expression of NOS isomers was the same in the placebo group sample.

In vitro animal studies (Table 4)

Also in animal models, morphological, biomechanical, and immunohistochemical evaluations have demonstrated how the synthesis of NO is induced during the reparative phase, and basal low concentrations are present in normal tendons [20]. It is not clear which cells are responsible for NO overproduction. This induction occurs in the early stages of repair, which are markedly impaired when enzyme activity is inhibited.

The mRNA expression of all 3 NOS isoforms was assessed after surgery on Achilles tendons of male rats, showing significant increased levels of mRNA in healing tendon. Fibroblasts and macrophages are likely to be markedly involved in the production of $\mathrm{NO}$, containing all the 3 isoforms. bNOS, a known central nervous system mediator, has been found in fibroblasts and is involved in wound repair. iNOS mRNA increases 4 days after the injury and gradually decreases thereafter. The peak of eNOS is at 7 days from injury; bNOS reaches its peak 21 days after the injury, but basal levels of all three enzymes were present in the control animals [13].

Supply of NO through NO-paracetamol application had beneficial effects on rat Achilles tendon healing by improving the amount of collagen and the material properties of the healing constructs and promoting better collagen reorganization [21].

As pro-inflammatory cytokines are upregulated when oxidative or other stresses occur, the increased expressions of IL-18, IL-15, and IL-6 in supraspinatus tears induce to hypothesize that these cytokines may modulate apoptosis: IL-18 stimulates the production of NO and counteracts the action of IL-15 and IL-6 which are anti-apoptotic factors. All mentioned cytokines induce the production and release of reactive oxygen species which influence negatively the healing of the tendon and, supposedly, can exacerbate apoptosis in overload conditions. By activating cytokines, apoptotic factors, and oxygen free radicals, this process may result in the tendon rupture [18].

\section{Discussion}

Although vasodilators and vasoconstrictors factors, finely balanced, regulate the physiological function of the 


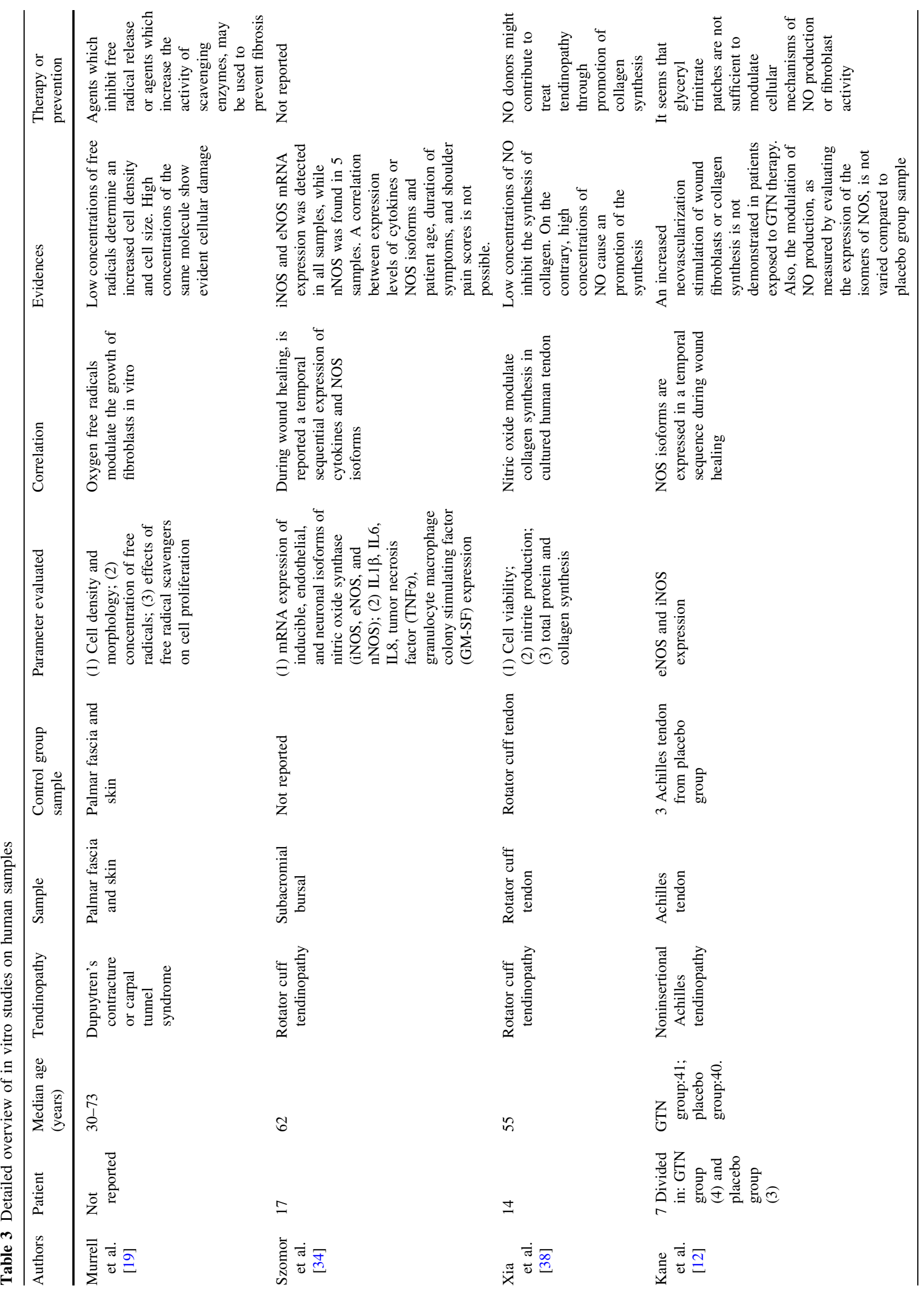




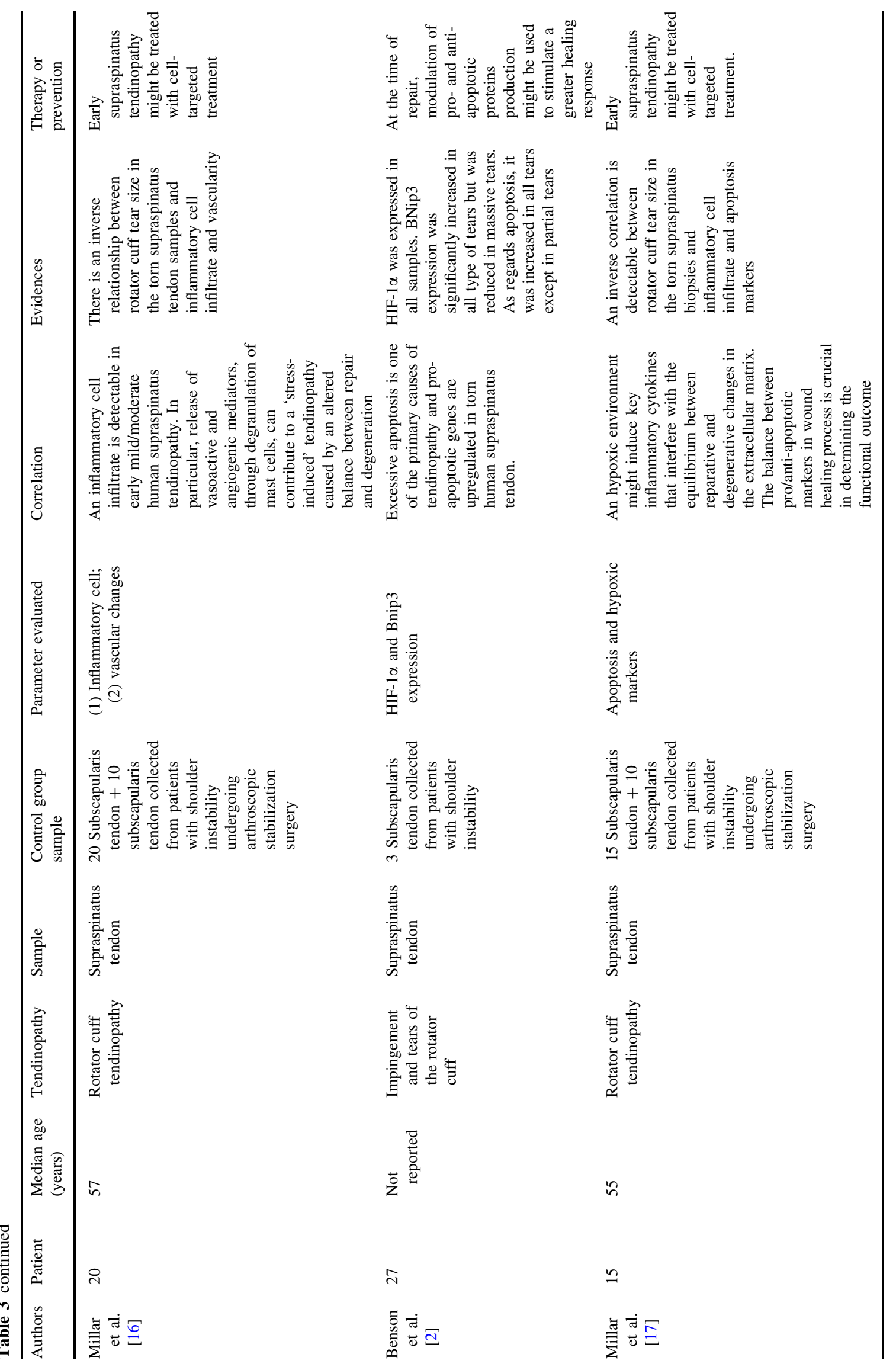




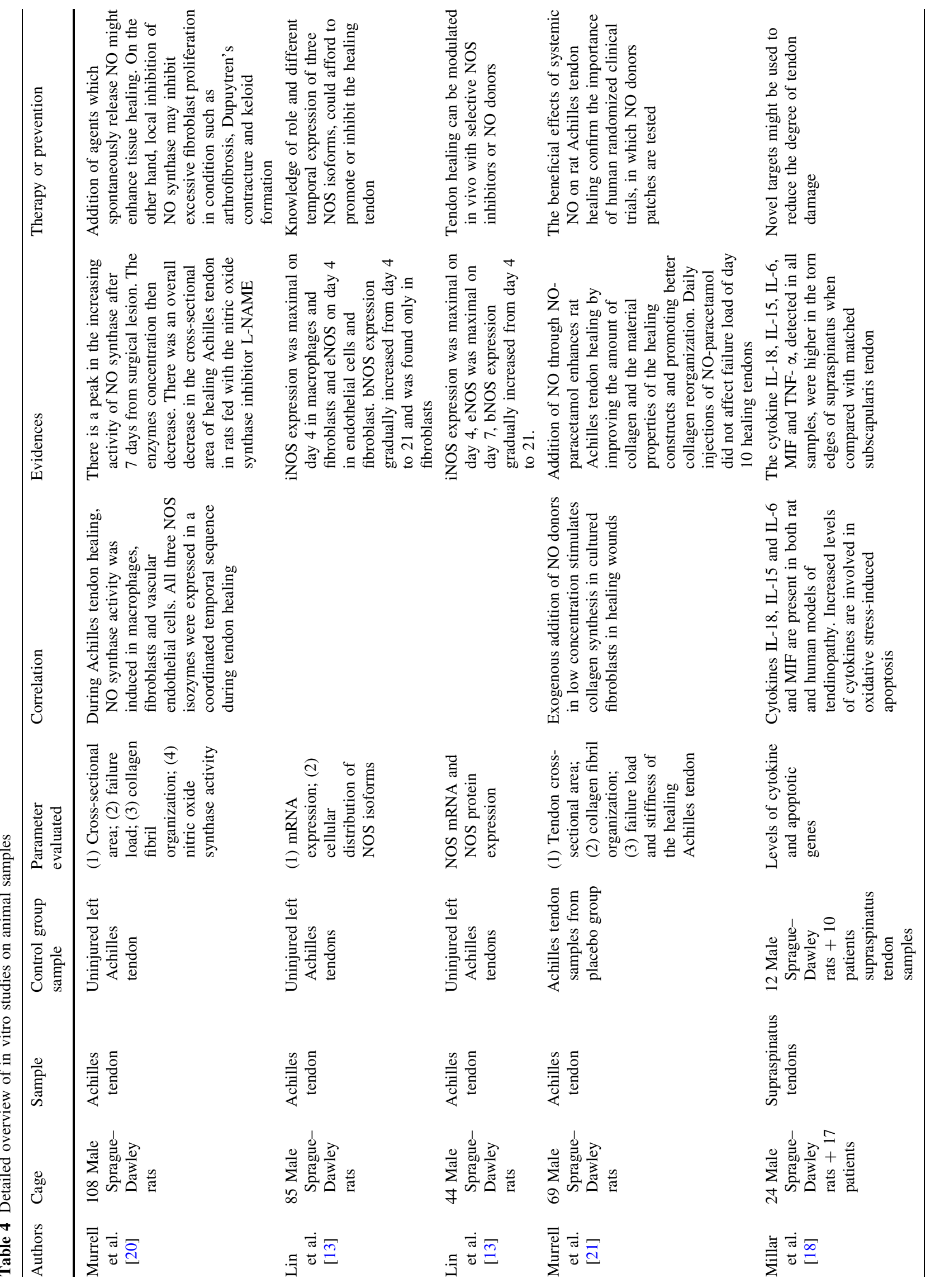


vascular bed, the mechanism of the endothelial dysfunction is still unknown. This condition is characterized by an inadequate response of the vessels to vasodilator agents, and high levels of vasoconstrictors such as angiotensin II, endothelin, and oxygen species (ROS) [32].

(1) Which factors are involved in endothelial dysfunction and what is the correlation between this condition and tendinopathy?

The production of NO, an endothelium-derived vasodilator, is induced when its concentration is low and when the balance between vasodilator and vasoconstrictors factors shifts in favor of the latter. This molecule prevents that subunits assemble themselves in a major enzyme which induces the release of ROS, and reacts against the factors produced by this enzyme. ROS is strictly involved in the development of this dysfunction, probably as mediator of apoptosis and inflammation. Similarly to what happens in hypoxia [17], ROS cause oxidative stress, overproduction of vasoconstrictors, and reduce the levels of NO [5]. As mechanical overload supposedly predispose to tendinopathy since it is able to induce vascular deregulation, but the molecular mechanisms underlying are still undefined. In this scenario, cell degeneration, related to increased activation of metalloproteinase and cellular apoptosis, and reparative cascades, with increased collagen synthesis and cell proliferation, coexist. NO induces repair and adaptation of the tendon tissues when they are exposed to physiological mechanical loads, whereas the over-expression of NOS isoforms which occurs when the tendon is overused is supposedly leading to a vicious circle with the final result of tendon degenerative changes [33], mediated by increased levels of metalloproteinase [6] and cytotoxicity [33]. Conversely, when the concentration of NO is low, angiogenesis and synthesis of collagen, tendon, bone, and cartilage repair are promoted $[20,26]$. The main site where tendinopathy occurs is therefore a hypovascularized area with decreased repair potential, often within the supraspinatus tendon [30]. Angiotensin II stimulates the production and release of reactive oxygen species such as ROS and NO from endothelial cells. Although endothelial dysfunction may predispose to cardiovascular disease, it is unclear what is the role that NO plays $[9,28]$.

(2) How is it possible to diagnose an endothelial dysfunction from bench to bedside?

To the best of the available knowledge, there are no indicative markers which make us able to diagnose this condition with absolute certainty. From the available evidence, NO could be diagnostic for endothelial disorders, but it is difficult to measure, as well as too expensive considering the eventual benefit that its measure could produce. However, it must be noted there are encouraging results reported on the adjuvant effect of the use of NO in the healing course of tendon lesions when combined with a rehabilitation treatment $[24,27,31]$.

(3) Can our understanding be helpful to treat and prevent cuff disorders?

Authors [38] reported that low doses of NO increase the synthesis of collagen in cultured human tendon cells. Paoloni et al. [26] showed that topical glyceryl trinitrate (GTN) application reduces pain and improves range of motion, force, and symptoms of shoulder impingement after 6 months of treatment, improving the outcomes of patients with chronic tendinopathy when combined with rehabilitation. Since there is no evidence yet that tendon pathology is directly related to endothelial dysfunction, although NO does improves clinical and functional outcomes compared to conventional treatments. The lack of specific randomized controlled trials and basic science studies does not allow us to understand whether endothelial dysfunction is important in the development of tendinopathy.

In conclusion, when present in low concentrations, $\mathrm{NO}$ works as a vasodilator agent and stimulator of local response in adaptation to mild stress conditions. At high concentrations, it is cause of tissue damage mediated by reactive oxygen species, activating specific molecular pathways $[19,38]$. Therefore, NO could be a marker to quantify the response of the endothelium to mechanical stress or hypoxia indicating the final balance between vasodilatating and vasoconstricting factors and their effects, but more ad stronger evidence is still needed to fully support this practice.

Conflict of interest None.

\section{References}

1. Abate M, Silbernagel KG, Siljeholm C, Di Iorio A, De Amicis D, Salini V, Werner S, Paganelli R (2009) Pathogenesis of tendinopathies: inflammation or degeneration? Arthritis Res Ther 11(3):235. doi:10.1186/ar2723

2. Benson RT, McDonnell SM, Knowles HJ, Rees JL, Carr AJ, Hulley PA (2010) Tendinopathy and tears of the rotator cuff are associated with hypoxia and apoptosis. J Bone Joint Surg $\mathrm{Br}$ 92(3):448-453. doi:10.1302/0301-620X.92B3.23074

3. Brooks CH, Revell WJ, Heatley FW (1992) A quantitative histological study of the vascularity of the rotator cuff tendon. J Bone Joint Surg Br 74(1):151-153

4. Castagna A, Cesari E, Gigante A, Conti M, Garofalo R (2013) Metalloproteases and their inhibitors are altered in both torn and intact rotator cuff tendons. Musculoskelet Surg 97(Suppl 1):39-47. doi:10.1007/s12306-013-0264-1 
5. Clapp BR, Hingorani AD, Kharbanda RK, Mohamed-Ali V, Stephens JW, Vallance P, MacAllister RJ (2004) Inflammationinduced endothelial dysfunction involves reduced nitric oxide bioavailability and increased oxidant stress. Cardiovasc Res 64(1):172-178. doi:10.1016/j.cardiores.2004.06.020

6. Garofalo R, Cesari E, Vinci E, Castagna A (2011) Role of metalloproteinases in rotator cuff tear. Sports Med Arthrosc 19(3):207-212. doi:10.1097/JSA.0b013e318227b07b

7. Garofalo R, Conti M, Massazza G, Cesari E, Vinci E, Castagna A (2011) Subcoracoid impingement syndrome: a painful shoulder condition related to different pathologic factors. Musculoskelet Surg 95(Suppl 1):S25-S29. doi:10.1007/s12306-011-0142-7

8. Hashimoto T, Nobuhara K, Hamada T (2003) Pathologic evidence of degeneration as a primary cause of rotator cuff tear. Clin Orthop Relat Res 415:111-120. doi:10.1097/01.blo.0000092974. 12414.22

9. Heo KS, Fujiwara K, Abe J (2011) Disturbed-flow-mediated vascular reactive oxygen species induce endothelial dysfunction. Circ J 75(12):2722-2730

10. Hirase T, Node K (2012) Endothelial dysfunction as a cellular mechanism for vascular failure. Am J Physiol Heart Circ Physiol 302(3):H499-H505. doi:10.1152/ajpheart.00325.2011

11. Huang TF, Perry SM, Soslowsky LJ (2004) The effect of overuse activity on Achilles tendon in an animal model: a biomechanical study. Ann Biomed Eng 32(3):336-341

12. Kane TP, Ismail M, Calder JD (2008) Topical glyceryl trinitrate and noninsertional Achilles tendinopathy: a clinical and cellular investigation. Am J Sports Med 36(6):1160-1163. doi:10.1177/ 0363546508314423

13. Lin J, Wang MX, Wei A, Zhu W, Murrell GA (2001) The cell specific temporal expression of nitric oxide synthase isoforms during achilles tendon healing. Inflamm Res 50(10):515-522

14. Lollino N, Brunocilla PR, Poglio F, Vannini E, Lollino S, Lancia M (2012) Non-orthopaedic causes of shoulder pain: what the shoulder expert must remember. Musculoskelet Surg 96(Suppl 1):S63-S68. doi:10.1007/s12306-012-0192-5

15. McCallum SD, Paoloni JA, Murrell GA (2011) Five-year prospective comparison study of topical glyceryl trinitrate treatment of chronic lateral epicondylosis at the elbow. Br J Sports Med 45(5):416-420. doi:10.1136/bjsm.2009.061002

16. Millar NL, Hueber AJ, Reilly JH, Xu Y, Fazzi UG, Murrell GA, McInnes IB (2010) Inflammation is present in early human tendinopathy. Am J Sports Med 38(10):2085-2091. doi:10.1177/ 0363546510372613

17. Millar NL, Reilly JH, Kerr SC, Campbell AL, Little KJ, Leach WJ, Rooney BP, Murrell GA, McInnes IB (2012) Hypoxia: a critical regulator of early human tendinopathy. Ann Rheum Dis 71(2):302-310. doi:10.1136/ard.2011.154229

18. Millar NL, Wei AQ, Molloy TJ, Bonar F, Murrell GA (2009) Cytokines and apoptosis in supraspinatus tendinopathy. J Bone Joint Surg Br 91(3):417-424. doi:10.1302/0301-620X.91B3.21652

19. Murrell GA, Francis MJ, Bromley L (1990) Modulation of fibroblast proliferation by oxygen free radicals. Biochem $\mathrm{J}$ 265(3):659-665

20. Murrell GA, Szabo C, Hannafin JA, Jang D, Dolan MM, Deng XH, Murrell DF, Warren RF (1997) Modulation of tendon healing by nitric oxide. Inflamm Res 46(1):19-27

21. Murrell GA, Tang G, Appleyard RC, del Soldato P, Wang MX (2008) Addition of nitric oxide through nitric oxide-paracetamol enhances healing rat achilles tendon. Clin Orthop Relat Res 466(7):1618-1624. doi:10.1007/s11999-008-0271-y

22. Notarnicola A, Maccagnano G, Di Leo M, Tafuri S, Moretti B (2013) Overload and neovascularization of Achilles tendons in young artistic and rhythmic gymnasts compared with controls: an observational study. Musculoskelet Surg. doi:10.1007/s12306013-0275-y
23. Oh LS, Wolf BR, Hall MP, Levy BA, Marx RG (2007) Indications for rotator cuff repair: a systematic review. Clin Orthop Relat Res 455:52-63. doi:10.1097/BLO.0b013e31802fc175

24. Paoloni JA, Appleyard RC, Nelson J, Murrell GA (2003) Topical nitric oxide application in the treatment of chronic extensor tendinosis at the elbow: a randomized, double-blinded, placebocontrolled clinical trial. Am J Sports Med 31(6):915-920

25. Paoloni JA, Appleyard RC, Nelson J, Murrell GA (2004) Topical glyceryl trinitrate treatment of chronic noninsertional achilles tendinopathy. A randomized, double-blind, placebo-controlled trial. J Bone Joint Surg Am 86-A(5):916-922

26. Paoloni JA, Appleyard RC, Nelson J, Murrell GA (2005) Topical glyceryl trinitrate application in the treatment of chronic supraspinatus tendinopathy: a randomized, double-blinded, placebocontrolled clinical trial. Am J Sports Med 33(6):806-813. doi:10. 1177/0363546504270998

27. Paoloni JA, Murrell GA, Burch RM, Ang RY (2009) Randomised, double-blind, placebo-controlled clinical trial of a new topical glyceryl trinitrate patch for chronic lateral epicondylosis. Br J Sports Med 43(4):299-302. doi:10.1136/bjsm.2008.053108

28. Pechanova O, Simko F (2007) The role of nitric oxide in the maintenance of vasoactive balance. Physiol Res 56(Suppl 2):S7S16

29. Randelli P, Spennacchio P, Ragone V, Arrigoni P, Casella A, Cabitza P (2012) Complications associated with arthroscopic rotator cuff repair: a literature review. Musculoskelet Surg 96(1):9-16. doi:10.1007/s12306-011-0175-y

30. Seitz AL, McClure PW, Finucane S, Boardman ND III, Michener LA (2011) Mechanisms of rotator cuff tendinopathy: intrinsic, extrinsic, or both? Clin Biomech (Bristol, Avon) 26(1):1-12. doi:10.1016/j.clinbiomech.2010.08.001

31. Steunebrink M, Zwerver J, Brandsema R, Groenenboom P, Akker-Scheek I, Weir A (2013) Topical glyceryl trinitrate treatment of chronic patellar tendinopathy: a randomised, double-blind, placebo-controlled clinical trial. Br J Sports Med 47(1):34-39. doi:10.1136/bjsports-2012-091115

32. Stevenson JH, Trojian T (2002) Evaluation of shoulder pain. J Fam Pract 51(7):605-611

33. Szomor ZL, Appleyard RC, Murrell GA (2006) Overexpression of nitric oxide synthases in tendon overuse. J Orthop Res 24(1):80-86. doi:10.1002/jor.20009

34. Szomor ZL, Wang MX, Kruller A, Murrell GA, Farmer KM, Kirkham BW, Bonar F (2001) Differential expression of cytokines and nitric oxide synthase isoforms in human rotator cuff bursae. Ann Rheum Dis 60(4):431-432

35. Thevendran G, Sarraf KM, Patel NK, Sadri A, Rosenfeld P (2013) The ruptured Achilles tendon: a current overview from biology of rupture to treatment. Musculoskelet Surg 97(1):9-20. doi:10.1007/s12306-013-0251-6

36. Thornton GM, Shao X, Chung M, Sciore P, Boorman RS, Hart DA, Lo IK (2010) Changes in mechanical loading lead to tendonspecific alterations in MMP and TIMP expression: influence of stress deprivation and intermittent cyclic hydrostatic compression on rat supraspinatus and Achilles tendons. Br J Sports Med 44(10):698-703. doi:10.1136/bjsm.2008.050575

37. Widlansky ME, Gokce N, Keaney JF Jr, Vita JA (2003) The clinical implications of endothelial dysfunction. J Am Coll Cardiol 42(7):1149-1160

38. Xia W, Szomor Z, Wang Y, Murrell GA (2006) Nitric oxide enhances collagen synthesis in cultured human tendon cells. J Orthop Res 24(2):159-172. doi:10.1002/jor.20060

39. Xia W, Wang Y, Appleyard RC, Smythe GA, Murrell GA (2006) Spontaneous recovery of injured Achilles tendon in inducible nitric oxide synthase gene knockout mice. Inflamm Res 55(1):40-45. doi:10.1007/s00011-005-0006-4 